\title{
The Proof of the Riemann Hypothesis and an Application to Physics
}

\author{
Rensley Meulens \\ Faculty of Engineering, University of Curaçao, Curaçao, Dutch Caribbean \\ Email: rensleymeulens@gmail.com
}

How to cite this paper: Meulens, R. (2019) The Proof of the Riemann Hypothesis and an Application to Physics. Applied Mathematics, 10, 967-988.

https://doi.org/10.4236/am.2019.1011068

Received: September 17, 2019

Accepted: November 17, 2019

Published: November 20, 2019

Copyright $\odot 2019$ by author(s) and Scientific Research Publishing Inc. This work is licensed under the Creative Commons Attribution International License (CC BY 4.0).

http://creativecommons.org/licenses/by/4.0/

\begin{abstract}
In this manuscript, a proof for the age-old Riemann hypothesis is delivered, interpreting the Riemann Zeta function as an analytical signal, and using a signal analyzing affine model used in radar technology to match the warped Riemann Zeta function on the time domain with its conjugate pair on the warped frequency domain (a Dirichlet series), through a scale invariant composite Mellin transform. As an application of above, since the Navier Stokes system solution's Dirichlet transforms are also Dirichlet series, a minimal general solution of the $3 \mathrm{~d}$ Navier Stokes differential equation for viscid incompressible flows is constructed through a fractional derivative Fourier transform of the found begin-solutions preserving the geometric properties of the $2 \mathrm{~d}$ version assuming that the solution is an analytic solution that suffices the Laplace equation in cylindrical coordinates, which is the momentum equation for both the $2 \mathrm{~d}$ and the $3 \mathrm{~d}$ Navier Stokes systems of differential equations.
\end{abstract}

\section{Keywords}

Riemann Hypothesis, Applied Mathematics, 3d Navier Stokes, Mathematical Physics, Dirichlet Transform

\section{Introduction}

The age-old Riemann Hypothesis is a conjecture that the Riemann Zeta function has its zeros only at the negative even integers and complex numbers with real part $\frac{1}{2}$ and is firstly stated in the essay by Bernard Riemann [1].

The fact that the (warped) Riemann Zeta function $\zeta\left(\frac{1}{2}+i v\right)$ under certain conditions will arise as the solution of a second order ordinary differential equation on the time domain while the Riemann Zeta function $\zeta(1-v)$ may arise as the same solution but on the warped frequency domain (domain of the $\mathrm{Di}$ - 
richlet transform (93) image functions defined originally on a time domain) gave rise to possible presumptions that the two mentioned functions may be a Dirichlet Transform of each other. Since the extended form of the Riemann Zeta function is found to be an eigenfunction of the Fourier Transform operator, a composite Mellin Transform is found that projects $\zeta\left(\frac{1}{2}+2 \pi i v\right)$ into $\zeta(1-v)$ preserving scale properties and is de facto a line-invariant transform between $\zeta\left(\frac{1}{2}+2 \pi i v\right)$ and $\zeta(v)$, since there exists a well-known functional relationship between $\zeta(1-v)$ and $\zeta(v)$, the so-called functional equation of the extended Riemann Zeta function

$$
\varsigma(s)=2^{s} \pi^{s-1} \sin \left(\frac{\pi s}{2}\right) \Gamma(1-s) \varsigma(1-s)
$$

A fact that confirms the age-old conjecture of Bernard Riemann.

The $C^{\infty}$-form of the eigenfunctions of the Fourier transform and the Dilation operator are thoroughly studied in [2]. The discrete and collapsed forms of these hyperbolic (eigen)functions are used as analytical input signals in [3] [4] [5] [6] [7] for an analytical signal analyzing affine model.

In this manuscript, the discrete super-position of the mentioned (warped) eigenfunctions are used to find a new eigenfunction of the Fourier Transform and to build solutions of the known and studied second order Ordinary Differential Equations (ODEs) on both time and warped frequency domain.

The solutions of the momentum equation of the Navier Stokes for both $2 \mathrm{~d}$ and $3 d$ versions are related to Dirichlet series (95), on the warped frequency domain. An appropriate inverse Dirichlet transform may be used to retrieve the solution on the time domain.

A general solution of the Navier Stokes $3 \mathrm{~d}$ equation for the viscid incompressible flows is constructed from the begin conditions in the warped frequency domain, using geometric properties of the $2 \mathrm{~d}$ version. This solution is called a minimal solution to the $3 \mathrm{~d}$ Navier Stokes equation for the viscid incompressible flows. Those types of functions may contain domain singularities, which will cause the blow-up time when the numerical pivots strike a singularity. Please do see Appendix A for a study of the Navier Stokes d.e.

\section{The Euler Differential Equation and a Think Experiment}

Consider the solutions for the Euler differential equation

$$
t^{2} y^{\prime \prime}+a t y^{\prime}+b y=0
$$

that may be deducted from the second order polynomial characteristic equation

$$
r^{2}+(a-1) r+b=0
$$

[With substitution of $t \frac{\mathrm{d} y}{\mathrm{~d} t}=\frac{\mathrm{d} y}{\mathrm{~d} x}$ to eliminate the non-constant terms does $t^{2} y^{\prime \prime}+a t y^{\prime}+b y=0$ transpose to $y^{\prime \prime}-(a-1) y^{\prime}+b=0$ and followed by the subs- 
titution of $y(t)=\mathrm{e}^{r x}$ delivers the found characteristic polynomial Equation (3).]

The solutions of the Euler differential equation [8] is given by

$$
y= \begin{cases}c_{1}|t|^{r_{1}}+c_{2}|t|^{r_{2}} & \text { for } D>0 \\ \left(c_{1}+c_{2} \ln |t|\right)|t|^{\alpha} & \text { for } D=0 \\ {\left[c_{1} \cos (\beta \ln |t|)+c_{2} \sin (\beta \ln |t|)\right]|t|^{\alpha}} & \text { for } D<0\end{cases}
$$

With

$$
\left\{\begin{array}{l}
\alpha=\frac{1}{2}(1-a) \\
\beta=\frac{1}{2} \sqrt{D}
\end{array}\right.
$$

and $D$ the discriminant equivalent with

$$
(a-1)^{2}-4 b
$$

If we think the variable $t$ as restricted to the set of natural numbers $\mathbb{N}$, and $c_{1}$ and $c_{2}$ as constants and equal to one and take improper super-position of all identified solutions, then the Riemann Zeta Function will arise as the family bundle of solutions of (2) as follows:

$$
y= \begin{cases}c_{1} \zeta(-\alpha-\beta)+c_{2} \zeta(-\alpha+\beta) & \text { for } D>0 \\ c_{1} \zeta(-\alpha)-c_{2} \zeta^{\prime}(-\beta) & \text { for } D=0 \\ \zeta(-\alpha+i \beta) & \text { for } D<0\end{cases}
$$

with the zeros of the characteristic polynomial (3) defined as $r_{1,2}=\alpha \pm \beta$ for

$$
D \geq 0
$$

and as $r_{1,2}=\alpha \pm i \beta$ when

$$
D<0
$$

In our think experiment we have got both the Riemann Zeta function $\zeta(-\alpha-\beta)$ and the warped Riemann Zeta function $\zeta(-\alpha+i \beta)$ and the derivative of the Riemann Zeta function $\zeta^{\prime}(-\beta)$ as solutions to the Equation (2).

The Riemann Zeta function arises also as a special case of the super-position of the general solutions (see Table B1 for examples in Appendix B) of other second order ordinary differential equations like the confluent hyper geometric differential equation called hyper geometric function of the first and second kinds, generalized Laguerre d.e., the Bessel d.e. etc. and the incompressible Navier-stokes equation

$$
\frac{\mathrm{d} \boldsymbol{u}}{\mathrm{d} t}+(\boldsymbol{u} \cdot \nabla) \boldsymbol{u}-v \Delta \boldsymbol{u}=\nabla \boldsymbol{p}+\boldsymbol{g}
$$

[9], but in the warped frequency domain. We would have expected then that the Riemann Zeta functions $\zeta\left(\frac{1}{2}+i v\right), \zeta( \pm v)$ would therefore reside on the warped frequency domain (as an image of the Dirichlet Transform operator). This is very fundamental in the sense that with proper assumptions and a proper 
time-frequency transformation these types of functions can co-exist both on the time and warped frequency domain as we are going to see next. Therefore it was very important for us to analyze the behavior of such functions on the warped frequency domain. Appendix B consists of a study of the relationship between discrete solutions of selected $2^{\text {nd }}$ order ODEs and the Riemann Zeta Function. Eigenfunctions of the Fourier Transform and the Dilation operators are thoroughly discussed in the thesis of Garas in [2] and the denomination "warped frequency domain" that is used in this manuscript is adapted from [2].

The Mellin transform operator [10] [11] does consist of the warping operator $U_{x}$, [2] p. 122 and further, followed by a Fourier transform. Its image will therefore reside on the warped frequency domain, when the initial function does reside on the time domain.

\section{The Extended Riemann Zeta Function as an Eigenfunction of the Fourier Transform}

Consider the monomial represented by $|x|^{\alpha}$ and its defined unitary Fourier transform (with ordinary frequency) $-2 \sin \left(\frac{\pi \alpha}{2}\right) \Gamma(1+\alpha)|2 \pi \nu|^{-\alpha-1}$. If we take the improper sum of both $|x|^{\alpha}$ and its Fourier Transform, a procedure also known as the Poisson Summation formula, then will the extended form of the Riemann Zeta function in the complex plane emerge as follows,

$$
\mathcal{F} \sum_{|x|=1}^{|x|=\infty}|x|^{\alpha}=\sum_{|v|=1}^{|v|=\infty}-2 \sin \left(\frac{\pi \alpha}{2}\right) \Gamma(1+\alpha)|2 \pi \nu|^{-\alpha-1}=\varsigma(-\alpha)
$$

using the functional equation for the extended Riemann Zeta function (1). In other words the extended Riemann Zeta function is invariant under the Fourier Transform.

This is a very important find because we can now derive a general formula for the Mellin Transform with fractional derivatives of the Fourier Transform and use it to calculate the Mellin Transform of the Riemann Zeta function.

Hence

$$
\mathcal{M}_{f}^{\xi}[\mathcal{X}](\beta)=\int_{0}^{\infty} \mathcal{X}(f) \mathrm{e}^{2 \pi i \xi f} f^{2 \pi i \beta+r} \mathrm{~d} f=\int_{-\infty}^{\infty} \mathcal{X}(f) \mathrm{e}^{2 \pi i \xi f} f^{2 \pi i \beta+r} \mathrm{~d} f
$$

[6] if all frequency components of $\mathcal{X}(f)$ are positive (or if $\mathcal{X}(f)$ is an analytical signal), can be seen as a Fourier Transform with transform variable $-|\xi|$ and with

$$
\mathcal{M}_{f}^{\xi=0}[\mathcal{X}](\beta)=\mathcal{M}_{f}[\mathcal{X}(f)](\beta)
$$

Then is

$$
\begin{gathered}
\mathcal{M}_{f}^{|\xi|}[\mathcal{X}](\beta)=\frac{i^{\mu}}{2 \pi} \frac{\partial^{\mu} \mathcal{F}[\mathcal{X}](f)(|\xi|)}{\partial|\xi|^{\mu}} \\
\text { with } \mathcal{F}[\mathcal{X}](f)(\xi)=\int_{-\infty}^{\infty} \mathcal{X}(f) \mathrm{e}^{-2 \pi i \xi f} \mathrm{~d} f
\end{gathered}
$$

and using the formula for the Fourier Transform of the $\mu$-th derivative of a 
Fourier integrable function $f$,

$$
\mathcal{F} t^{\mu} f(t)(\xi)=i^{\mu} \frac{\partial^{\mu} \mathcal{F}[f](t)(\xi)}{\partial \xi^{\mu}}
$$

with $i$ the imaginary unit and $\mu \in \mathbb{C}$ extended from the set of natural numbers $\mathbb{N}$ to the set of complex numbers $\mathbb{C}$ and with

$$
\mu=2 \pi i \beta+r
$$

If we take the Riemann Zeta function as the analytical signal

$$
\mathcal{X}(f)=\sum_{|f|=1}^{|f|=\infty}|f|^{-\alpha}
$$

will above formula yield for

$$
\begin{aligned}
& \mathcal{M}_{f}^{-\xi}[\mathcal{X}](\beta)=\int_{-\infty}^{\infty} \sum_{|f|=1}^{|f|=\infty}|f|^{-\alpha} \mathrm{e}^{-2 \pi i \xi f} f^{2 \pi i \beta+r} \mathrm{~d} f \\
& =\frac{i^{\mu}}{2 \pi} \frac{\partial^{\mu} \mathcal{F} \sum_{|f|=1}^{|f|=\infty}|f|^{-\alpha}(\xi)}{\partial \xi^{\mu}} \\
& =\frac{i^{\mu}}{2 \pi} \mathcal{F} \frac{\partial^{\mu} \sum_{|f|=1}^{|f|=\infty}|f|^{-\alpha}(\xi)}{\partial f^{\mu}} \\
& =\mathcal{F} \sum_{|f|=1}^{|f|=\infty} \overbrace{2 \pi i^{-\mu} \frac{\Gamma(\mu+\alpha)}{\Gamma(\alpha)} \xi}^{\text {scaling effect }} \xi^{-\mu-\alpha} \\
& =2 \pi i^{-\mu} \frac{\Gamma(\mu+\alpha)}{\Gamma(\alpha)} \varsigma(\mu+\alpha)
\end{aligned}
$$

with $\mu=2 \pi i \beta+r$ using the fact that the fractional derivative of

$$
\frac{\partial^{\mu} x^{-\alpha}}{\partial x^{\mu}}=-1^{\mu} \frac{\Gamma(\mu+\alpha)}{\Gamma(\alpha)} x^{-(\alpha+\mu)}
$$

for $\alpha \geq 0$ and $\xi \geq 0$ [7]. The identified scaling and dilative effect (or Doppler effect) makes this transform ideal for analytical signal reconstruction and signal analysis purposes applied in radar technology.

\section{The Proof of the Age-Old Riemann Hypothesis}

For normalization purposes and to be coherent with the used Fourier transform we will use $\zeta\left(\frac{1}{2}+2 \pi i v\right)$ instead of $\zeta\left(\frac{1}{2}+i v\right)$.

An affine or a line-preserving map between $\zeta\left(\frac{1}{2}+2 \pi i v\right)$ and $\zeta(1-s)$ may be formed by the composite Mellin transform operator $\mathcal{M}_{v} \mathcal{M}_{n}$, with

$$
\mathcal{M}_{n} f=\mathcal{F} U_{n} f=\mathcal{F} \mathrm{e}^{-\frac{n}{2}} f\left(\mathrm{e}^{n}\right)
$$

and $U_{n}$ the warping operator on variable $n$ and $\mathcal{F}$ the Fourier transform. ( $\mathcal{M}_{n}$, similarly with the discrete Mellin Transform used in [6], entails a manipulation with the summation index variable to calculate the result). Using the found composite Mellin transform on $\zeta\left(\frac{1}{2}+2 \pi i v\right)$ will yield 


$$
\begin{aligned}
& \mathcal{M}_{v} \mathcal{M}_{n} \zeta\left(\frac{1}{2}+2 \pi i v\right) \\
& =\mathcal{M}_{v} U_{n} \mathcal{F} \zeta\left(\frac{1}{2}+2 \pi i v\right)=\mathcal{M}_{v} \mathcal{F} \sum_{n=1}^{n=\infty} \mathrm{e}^{-\frac{n}{2}} \frac{\mathrm{e}^{-2 \pi i v \log \left(\mathrm{e}^{-n}\right)}}{\sqrt{\mathrm{e}^{-n}}} \\
& =\mathcal{M}_{v} \mathcal{F} \sum_{n=1}^{n=\infty} \mathrm{e}^{2 \pi i v n}=\mathcal{M}_{v} \sum_{n=1}^{n=\infty} \delta(v-n)=\zeta(1-s)
\end{aligned}
$$

Q.e.d. The "quod erat demonstrandum" abbreviation refers to the confirmation of the age-old Riemann Hypothesis, knowing that there exists a functional equation between $\zeta(1-s)$ and $\zeta(s)$.

The popularized expression for the Riemann Zeta function in the complex plane on the so-called critical line, $\zeta\left(\frac{1}{2}+i s\right)$, is also a solution of higher order ODEs when the pair of complex conjugate solutions of the corresponding characteristic polynomials are repeating as we have seen in our thought experiment. Its warped version (thus warping of a warped function with $\mathcal{M}^{2}$ ) can be represented as an even characteristic polynomial, since the Riemann Zeta function is an eigenfunction of the Fourier transform. Hence

$$
\begin{aligned}
& \mathcal{M}_{n} \zeta\left(\frac{1}{2}+2 \pi i v\right) \stackrel{\text { using (22) }}{=} \mathcal{M}_{s}^{-1}\{\zeta(1-s)\}=2 \sum_{v=1}^{v=\infty} \cos (v 2 \pi x) \\
& =2 \sum_{v=1}^{v=\infty} T_{v}(\cos (2 \pi x))=\lim _{v \rightarrow \infty} U_{2 v}(\cos \pi x)-1 \\
& =a_{2 v} \tilde{x}^{2 v}+a_{2 v-2} \tilde{x}^{2 v-2}+\cdots+a_{2} \tilde{x}^{2}-1
\end{aligned}
$$

and with $\tilde{x}=\cos \pi x$, with $a_{2 v}$ defined as in the On-Line Encyclopedia of Integer Sequences as OAEIS A053117 [13], that is comparable with a characteristic polynomial of a higher order ODE for some $v \rightarrow \infty$. Here are $T_{v}$ and respectively $U_{v}$ the Chebyshev polynomials of resp. the first and second kinds [14]. Since the index $v$ has to be taken even in the cumulative $U_{v}$ polynomial gives the opportunity to form repeating pairs of conjugate complex roots as the resulting characteristic polynomial may then be decomposed in product of lower order parabolic expressions which is a conditio sine qua non for the warped Riemann Zeta function to exist as a solution of the discussed ODE.

The expression $-2 \sum_{v=1}^{v=\infty} \cos (2 \pi x v)$ is equivalent with the Fourier series of the zero-th Bernoulli polynomials,

$$
B_{n}(x)=\frac{n !}{2 \pi i^{n}} \sum_{v \neq 0} \frac{\mathrm{e}^{2 \pi i v x}}{v^{n}}=-2 n ! \sum_{v \in \mathbb{N} /\{0\}} \frac{\cos \left(2 \pi x v-\frac{n \pi}{2}\right)}{2 v \pi^{n}}
$$

The last definition can be found in the public accessible on-line Wikipedia.

If we do interchange the variable $x$ and $n$ with each other, a logic way to calculate the Dirichlet Transform of an Ordinary Generating Function resulting in a Dirichlet series, yields:

$$
\begin{gathered}
B_{x}(n)=-2^{1-x} \pi^{-x} x ! \sum_{v=1}^{v=\infty} k^{-x} \sin \left(\frac{\pi}{2}[1-4 v n+x]\right) \text { for } \\
0 \leq x \leq 1
\end{gathered}
$$


what equivalent is with $-\zeta(1-s)$ for $n=0$ and is called the Hurwitz formula for the generalized extended zeta function $\zeta(x, n)$ and with $n \in \mathbb{Z}$ the wellknown functional equation for the Riemann Zeta function will emerge.

The extended generalized zeta function is invariant under the Dirichlet Transform (and so under the Mellin Transform), when transforming only the domain of its arguments will undergo a variable swap as we have seen. The Hurwitz Zeta function is therefore an eigenfunction under both the Dirichlet and the Mellin transforms.

$$
\begin{aligned}
& \overbrace{\zeta\left(\frac{1}{2}+2 \pi i v\right) \rightarrow 2 \sum_{v=1}^{v=\infty} T_{v}(\cos (2 \pi y)) \equiv \underbrace{\lim _{v \rightarrow \infty} U_{2 v}(\cos \pi y)-1}_{=\sum_{v>0} a_{2} \tilde{x}^{\tilde{x}^{2}}-1 ?}}^{\mathcal{M}_{x}} \\
& \text { basic Dirichlet series } \\
& \underbrace{2 \sum_{v=1}^{v=\infty} T_{v}(\cos (2 \pi y)) \equiv \lim _{v \rightarrow \infty} U_{2 v}(\cos \pi y)-1 \rightarrow \overbrace{\zeta(1-s)}^{\text {with negative argument }}}_{\mathcal{M}_{y}}
\end{aligned}
$$

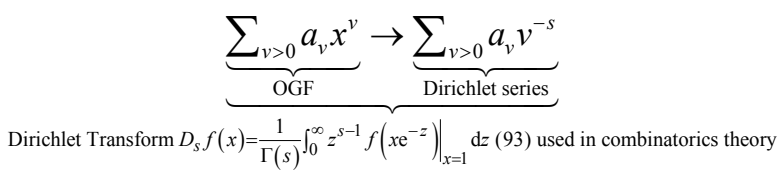

[15] p. 177.

\section{Conclusions and Future Work}

Using the fact that the extended Riemann Zeta function is an eigenfunction under the Fourier Transform, it is proved that there exists a line-invariant composite Mellin transform operator that projects $\zeta\left(\frac{1}{2}+2 \pi i x\right)$ into $\zeta(1-s)$ namely

$$
\mathcal{M}_{x} \mathcal{M}_{n} \zeta\left(\frac{1}{2}+2 \pi i x\right)=\zeta(1-s)
$$

what confirms the validation of the age-old Riemann Hypothesis, knowing that there exists a well-known functional equation between $\zeta(1-s)$ and $\zeta(s)$.

De facto they are conjugate pairs of each other using repetitively a fractional derivative Fourier Transform operator or the so-called Mellin Transform operator $\mathcal{M}_{f}^{\xi}$ (12). The Mellin transform will expand the transform variable for $\mathcal{M}_{n}$ from the set of natural numbers $\mathbb{N}$ to the set of real numbers $\mathbb{R}$ and $\mathcal{M}_{x}$ will expand the transform variable from the set of real numbers to the set of (negative) complex numbers $\mathbb{C}$.

Our future work will entail the study of the full spectrum of the eigenspaces belonging to the (fractional derivative) Fourier Transform operator and the behavior of the so-called conjugate pairs on their respectively domains with regard to known solution procedures for partial differential equations.

\section{Conflicts of Interest}

The author declares no conflicts of interest regarding the publication of this paper. 


\section{References}

[1] Riemann, B. (1859) Ueber die Anzahl der Primzahlen Unter einen gegebenen Gröss. Monatsberichte der Berliner Akademie, 1-9.

[2] Garas, J. (1999) Adaptive 3D Sound Systems. Thesis, Technische Universiteit Eindhoven, Eindhoven. https://doi.org/10.1007/978-1-4419-8776-1

[3] Bertrand, J., Bertrand, P. and Ovarlez, J.P. (1990) Discrete Mellin Transform for Signal Analysis. Proceedings of ICASSP, Vol. 3, 1603-1606.

[4] Bertrand, J., Bertrand, P. and Ovarlez, J. (1989) Compression d'Impulsion en large bande. XIIieme Colloque sur le traitement du signal et des images, Paris, Vol. 12, 21-24.

[5] Ovarlez, J.P. (1991) Calcul des Fonctions D’ámbiguie Large Bande Par Transformee de Mellin. Proceedings of XIIIeme Coll. GRESTI, Chatilon Cedex.

[6] Ovarlez, J.P. (1994) La Transformation de Mellin Transform et L'analyse des signaux Large Bande. Coll. Temps-Frequence, Ondelettes et Multiresolution: Theorie, Modeles et Applications, Vol. 9, 1-8.

[7] Bertrand, J. and Bertrand, P. (1969) Wavelets Time Frequency Methods and PhaseSpace. Springer-Verlag, Berlin, 164-171.

[8] Wikipedia Contributors (2006) Cauchy-Euler Equation. https://en.wikipedia.org/wiki/Cauchy\%E2\%80\%93Euler_equation

[9] Powell, A. (2010) The Navier-Stokes Equations. ftp://texmex.mit.edu/pub/emanuel/CLASS/12.340/navier-stokes(2).pdf

[10] Poularikas, E. (1999) The Handbook of Formulas and Tables for Signal Processing. CRC Press, Boca Raton. https://doi.org/10.1201/9781420049701

[11] Poularikas, E. (2010) Transforms and Application Handbook. Third Edition, CRC Press, Boca Raton. https://doi.org/10.1201/9781420066531

[12] Wikipedia Contributors (2018) Fractional Calculus. https://en.wikipedia.org/wiki/Fractional_calculus

[13] OEIS A053117 (1964) The Online Encyclopedia of Integer Sequences. https://oeis.org/A053117

[14] Wikipedia Contributors (2017) Chebyshev Polynomials. http://en.wikipedia.org/wiki/Chebyshev_polynomials

[15] Melzak, Z.A. (2012) Companion to Concrete Mathematics. Dover Publications, Incorporated, Mineola.

[16] Wikipedia Contributors (2015) Heaviside Step Function. https://e.wikipedia.org/wiki/Heaviside_step_function

[17] Wikipedia Contributors (2008) Euler Product. https://en.wikipedia.org/wiki/euler_product

[18] Srinivasan, G.K. (2012) A Unified Approach to the Integrals of Mellin-BarnesHecke Type. https://arxiv.org/pdf/1208.6079.pdf

[19] Wikipedia Contributors (2018) Confluent Hypergeometric Function. https://en.wikipedia.org/w/index.php?title=Confluent_hypergeometric_function\&ol did=865747301

[20] Vojta, V. (2013) An Interconnection between Cayley-Eisenstein-Pólya and Landau Probability Distributions. Acta Polytechnica, 53, 63-69.

[21] Wikipedia Contributors (2015) The Associated Laguerre Polynomials. https://e.wikipedia.org/wiki/Lageurre_polynomials 
[22] Wikipedia Contributors (2010) Bessel Polynomials.

https://en.wikipedia.org/w/index.php?title=Bessel_polynomials\&oldid=882389474

[23] The Online Encyclopedia of Integer Sequences (1964) The Online Encyclopedia of Integer Sequences OIES A113025. https://oeis.org/A113025 


\section{Appendix A}

Minimal solution for the $3 \mathrm{~d}$ viscid incompressible Navier Stokes equation: an application to physics

The solution of the Laplace equation in cylindrical coordinates is of paramount importance in many applications and this is not an exception for finding a minimal solution of the $3 \mathrm{~d}$ Navier Stokes equations for the viscid incompressible media. The viscid flow arises when the viscous forces are prominent present in the equations. The solution is called minimal because a $2 \mathrm{~d}$ geometric solution is extended to the $3 \mathrm{~d}$ case, where domain singularities might be inherited.

If we extend the solutions to the complex space and take the super-positioned result of all positive discretized solutions will yield a polynomial or hyperbolic progression, which is not surprising as we are going to see.

Hence all second order ordinary differential equations that we have studied in this manuscript do have a polynomial progression as a solution as we are going the see in the next paragraphs.

The solution of the Mellin Transformed Laplace equation in cylindrical coordinates on the radius variable

$$
\begin{aligned}
& \left\{\begin{array}{l}
r^{2} f_{r r}+r f_{r}+r f_{\theta \theta}=0 \\
\frac{1}{r} \frac{\partial}{\partial r}\left(r \frac{\mathrm{d} f}{\mathrm{~d} r}\right)+\frac{1}{r^{2}} \frac{\partial^{2} f}{\partial \theta^{2}}=0 \\
F_{\theta \theta}+s^{2} F=0
\end{array}\right. \\
& \text { is } A(s) \cos (\theta s)+B(s) \sin (\theta s)
\end{aligned}
$$

If we discretize $\theta=n \in \mathbb{N}$, then take the superposition of all discrete solutions, is still a general solution of above equation, namely

$$
\sum_{1}^{\infty} F(s, n)=\sum_{1}^{\infty} C_{1}(s) \cos (s n)+C_{2}(s) \sin (s n)
$$

and using $2 \pi s$ instead of $s$ and taking $A(s)=1$ and $B(s)=-i$ (to simplify the expression), then will the solution become

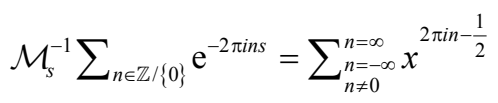

since $\sum_{n \in \mathbb{Z} /\{0\}} \mathrm{e}^{-2 \pi i n s}$ is invariant under the Fourier Transform or

$$
\sum_{|x|}|x|^{2 \pi i n-\frac{1}{2}}
$$

if we would have taken summation over the variable $x$.

If we take now the Dirichlet Transform of the solution (33) for $n>0$, will this result in $\varphi(s)$ with $\varphi(s)=f_{4}(s)=\sum_{n=1}^{n=\infty} a_{n} n^{-s} 2 \pi i^{-s}$ if we would have used a general term $a_{n}$ [15]. Please note that

$$
\begin{aligned}
& \varphi(s)=f_{4}(s)=\sum_{n=1}^{n=\infty} a_{n} n^{-s} 2 \pi i^{-s} \Gamma(s) \text { for } a_{n}=1, \text { yields } \\
& 2 \pi i^{-s} \Gamma(s) \varsigma(s)=\frac{\frac{i^{-s}}{2} \varsigma(1-s)}{\cos \left(\frac{\pi s}{2}\right)}=\frac{1}{2} \varsigma(1-s)-\frac{i}{2} \varsigma(1-s) \tan \left(\frac{\pi s}{2}\right)
\end{aligned}
$$




$$
=\varsigma(1-s)\left(\frac{1}{2}-\frac{i}{2} \tan \left(\frac{\pi s}{2}\right)\right) \cong \theta(-i s) \varsigma(1-s)
$$

with $\theta(s)$ the Heaviside step function [16].

Above, the so-called functional equation of the Riemann Zeta function (1) is used, and

$$
\left\{\begin{array}{l}
\Gamma(s) \Gamma(1-s)=\frac{\pi}{\sin (\pi s)} \\
\sin (\pi s)=\frac{1}{2} \cos \left(\frac{\pi s}{2}\right) \sin \left(\frac{\pi s}{2}\right) \\
\theta(s) \cong \frac{1}{2}+\frac{1}{2} \tanh \left(\frac{\pi s}{2}\right)= \begin{cases}1 & \text { for } s \rightarrow \infty \\
\frac{1}{2} & \text { for } s=0 \\
0 & \text { for } s \rightarrow-\infty\end{cases}
\end{array}\right.
$$

The Mellin Transform of (32), for $C_{1}(s)=f(s)$ and $C_{2}(s)=$ if $(s)$, may be represented as

$$
\begin{aligned}
& \mathcal{M}_{x}^{-|n|}\left[\sum_{1}^{\infty} F(x, n)\right](\mu)=\mathcal{M}_{x} \sum_{1}^{\infty} \mathrm{e}^{-i|n| x} f(\mu) \\
& \mathcal{M}_{x} \sum_{1}^{\infty} \mathrm{e}^{-i|n| x} f(x)(\mu)=\sum_{1}^{\infty} \int_{0}^{\infty} \mathrm{e}^{-i|n| x} f(x) x^{\mu} \mathrm{d} x \\
& =\int_{0}^{\infty} U \zeta\left(\frac{1}{2}+i x\right) \underbrace{f(x)}_{\text {taylor series expansion: }} x^{\mu} \mathrm{d} x \\
& =\sum_{k=0}^{k=\infty} \int_{0}^{\infty} U \zeta\left(\frac{1}{2}+i x\right) x^{\mu} f^{(k)}(0) \frac{x^{k}}{k !} \mathrm{d} x
\end{aligned}
$$

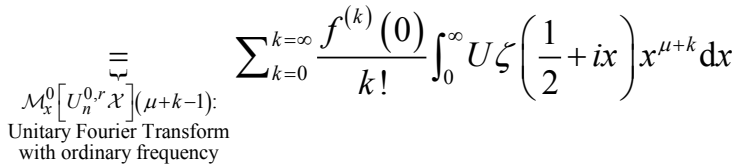

$$
\begin{aligned}
& =\sum_{k=0}^{k=\infty} \frac{f^{(k)}(0)}{k !} \int_{0}^{\infty} \mathrm{e}^{-2 \pi i n \mu} \zeta\left(\frac{1}{2}+i x\right) x^{\mu+k} \mathrm{~d} x \\
& =\sum_{k=0}^{k=\infty} \frac{i^{\mu+k}}{2 \pi} \frac{f^{(k)}(0)}{k !} \frac{\partial^{\mu+k}}{\partial n^{\mu+k}} \mathcal{F} \zeta\left(\frac{1}{2}+i x\right) \\
& =\sum_{k=0}^{k=\infty} \frac{i^{\mu+k}}{2 \pi} \frac{f^{(k)}(0)}{k !} \frac{\partial^{\mu+k}}{\partial n^{\mu+k}} \zeta\left(\frac{1}{2}+i x\right) \\
& =\sum_{k=0}^{k=\infty} 2 \pi i^{-\mu-k} f^{(k)}(0) \frac{\Gamma\left(i x+\mu+k+\frac{1}{2}\right)}{\Gamma(k+1) \Gamma\left(i x+\frac{1}{2}\right)} \zeta\left(i x+\mu+k+\frac{1}{2}\right) \\
& \text { with } \sum_{0}^{\infty} \frac{f^{(k)}(0)}{k !} x^{k}
\end{aligned}
$$

the series expansion of the begin condition $f$ around the value 0 . Equation (38) is de facto a solution on the warped frequency domain to the eigenvalue problem Equation (30) or the Helmholtz equation that typically arises in physical problems involving partial differential equations in both space and time (e.g. the viscid Navier Stokes incompressible d.e.). 
When the begin conditions differ per independent solution legs, the same method may be applied but now instead of using $\mathrm{e}^{-i|n| x}$, its real part (with regard to the term with $C_{1}(s)$ ) must be used and respectively its imaginary part (with regard to the term with $C_{2}(s)$ ) must be used to calculate (38).

The time domain equivalent of (38) may be calculated by the inverse Dirichlet transform and is defined by

$$
\begin{aligned}
\sum_{n=1}^{n=\infty} \sum_{k=0}^{k=\infty} 2 \pi i^{-\mu-k} f^{(k)}(0) & \frac{\Gamma(2 n+k)}{\Gamma(k+1) \Gamma(n) \Gamma(n)} x^{(-2 n-k)} \\
\text { taking } \mu & =i x+\frac{1}{2}
\end{aligned}
$$

The Dirichlet Transform of a function $f$ is defined as

$$
\frac{1}{\Gamma(\mu)} \mathcal{M} f\left(\mathrm{e}^{x}\right)=\frac{\mathrm{e}^{-2 \pi i \xi \mid x}}{\Gamma(\mu)} \mathcal{M} f\{\mu\}=\frac{1}{\Gamma(\mu)} \frac{i^{\mu}}{2 \pi} \frac{\partial^{\mu}}{\partial|\xi|^{\mu}} \mathcal{F}[f](\mu)(|\xi|)
$$

what is equivalent with

$$
\frac{1}{\Gamma(\mu)} \mathcal{M}_{x}^{-|\xi|} f(x)(\mu)
$$

The above solution (38) can also be used for the reconstruction of the general solution (for the velocity profile, and thus also for the pressure warped frequency distribution) of the incompressible 2d-Navier Stokes equation in cylinder coordinates, taking

$$
\begin{gathered}
\theta=n \\
\text { and } r=s
\end{gathered}
$$

since the Laplace equation in cylindrical coordinates is congruent with the $2 \mathrm{~d}$ momentum equation for an incompressible flow. The solution for the (warped) velocity distribution function is therefore:

$$
u(\mu)=\sum_{k=0}^{k=\infty} 2 \pi i^{-\mu-k} f^{(k)}(0) \frac{\Gamma\left(i x+\mu+k+\frac{1}{2}\right)}{\Gamma(k+1) \Gamma\left(i x+\frac{1}{2}\right)} \zeta\left(i x+\mu+k+\frac{1}{2}\right)
$$

With the begin function $f$ equivalent with 1 does Equation (46) yield the Dirichlet series $2 \pi i^{-\mu} \frac{\Gamma(2 \mu)}{\Gamma(\mu)} \varsigma(2 \mu)$ taking $\mu=i x+\frac{1}{2}$ :

$$
\left\{\begin{array}{l}
\mu=i x+\frac{1}{2} \\
2 \pi i^{-\mu} \frac{\Gamma(2 \mu)}{\Gamma(\mu)} \varsigma(2 \mu) \quad \text { warped frequency domain (see Figure A1) } \\
\sum_{n=1}^{n=\infty} 2 \pi i^{-n} \frac{\Gamma(2 n)}{\Gamma(n) \Gamma(n)} x^{-2 n}=-\frac{i \sqrt{\pi}}{2 x^{2}\left(\frac{\pi x^{2}+2 i}{x^{2}}\right)^{\frac{3}{2}}},|x|>\sqrt{\frac{2}{\pi}} \text { time domain (see Figure A2) }
\end{array}\right.
$$

Figure A1 confirms the volatile characteristics of the Dirichlet series. Figure A2 is the inverse Dirichlet transform of Figure A1, using [15]. 


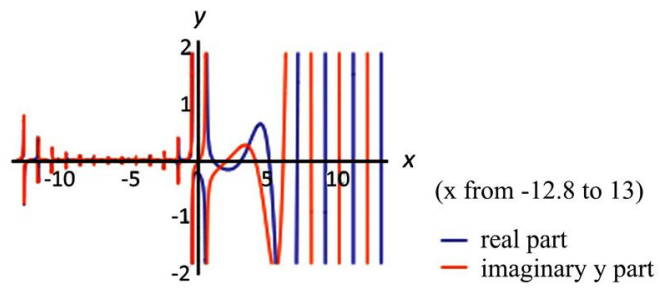

Figure A1. $u(\mu)$ warped frequency domain.

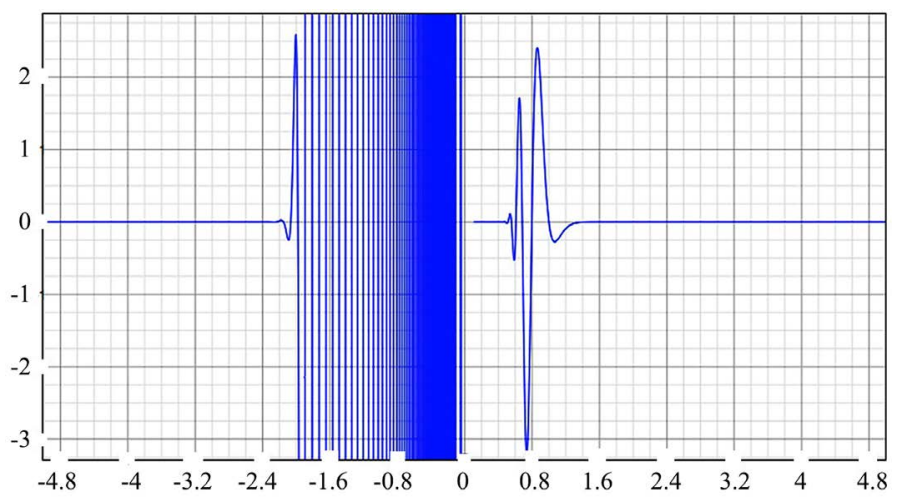

Figure A2. $u(x)$ time domain.

The 3d Navier Stokes system of equations for the incompressible viscid flow [9] is reducible to an extent that its general and exact solutions are determined by the (general and exact) solutions of the $2 \mathrm{~d}$ Laplace equation in cylindrical coordinates (as above) as follows. We will call this solution a minimal solution.

Consider the NVS 3d systems of equations for the incompressible viscid flow:

$$
\begin{gathered}
\rho \frac{\partial \boldsymbol{u}}{\partial t}+\rho(\boldsymbol{u} \cdot \nabla) \boldsymbol{u}-\mu \nabla^{2} \boldsymbol{u}=-\nabla \boldsymbol{p}+\rho \boldsymbol{g} \\
\text { and }\left\{\begin{array}{l}
\mu=\text { viscid constant } \\
\rho=\text { medium density }
\end{array}\right.
\end{gathered}
$$

Equation (48) per directional component in cylindrical coordinates:

$$
\begin{gathered}
\rho\left(\frac{\partial u_{r}}{\partial t}+u_{r} \frac{\partial u_{r}}{\partial r}+\frac{u_{\theta}}{r} \frac{\partial u_{r}}{\partial \theta}-\frac{u_{\theta}^{2}}{r}+u_{z} \frac{\partial u_{r}}{\partial z}\right) \\
=-\frac{\partial \boldsymbol{P}}{\partial r}+\rho g_{r}+\mu\left(\frac{1}{r} \frac{\partial}{\partial r}\left(r \frac{\partial u_{r}}{\partial r}\right)-\frac{u_{r}}{r^{2}}+\frac{1}{r^{2}} \frac{\partial^{2} u_{r}}{\partial \theta^{2}}-\frac{2}{r^{2}} \frac{\partial u_{\theta}}{\partial \theta}+\frac{\partial^{2} u_{r}}{\partial z^{2}}\right) \\
\rho\left(\frac{\partial u_{\theta}}{\partial t}+u_{r} \frac{\partial u_{\theta}}{\partial r}+\frac{u_{\theta}}{r} \frac{\partial u_{\theta}}{\partial \theta}+\frac{u_{\theta} u_{r}}{r}+u_{z} \frac{\partial u_{\theta}}{\partial z}\right) \\
=-\frac{\partial \boldsymbol{P}}{\partial \theta}+\rho g_{\theta}+\mu\left(\frac{1}{r} \frac{\partial}{\partial r}\left(r \frac{\partial u_{\theta}}{\partial r}\right)-\frac{u_{\theta}}{r^{2}}+\frac{1}{r^{2}} \frac{\partial^{2} u_{\theta}}{\partial \theta^{2}}+\frac{2}{r^{2}} \frac{\partial u_{r}}{\partial \theta}+\frac{\partial^{2} u_{\theta}}{\partial z^{2}}\right) \\
\rho\left(\frac{\partial u_{z}}{\partial t}+u_{r} \frac{\partial u_{z}}{\partial r}+\frac{u_{\theta}}{r} \frac{\partial u_{z}}{\partial \theta}+u_{z} \frac{\partial u_{z}}{\partial z}\right) \\
=-\frac{\partial \boldsymbol{P}}{\partial z}+\rho g_{z}+\mu\left(\frac{1}{r} \frac{\partial}{\partial r}\left(r \frac{\partial u_{z}}{\partial r}\right)+\frac{1}{r^{2}} \frac{\partial^{2} u_{z}}{\partial \theta^{2}}+\frac{\partial^{2} u_{z}}{\partial z^{2}}\right)
\end{gathered}
$$


To solve the above 3d NVS system of equation we will have to reduce the r.h.s. of each equation to the sum of the pressure term and the external force and equating the Laplace expression in cylindrical coordinates to zero (solve up the Laplacian to get the velocity distribution), and then expand this using time convolution to $u(\boldsymbol{x}, t)$ with the appropriate Gaussian diffusion Green's function to solve the enclosed diffusion equation

$$
\rho \frac{\partial \boldsymbol{u}}{\partial t}=\mu \nabla^{2} \boldsymbol{u}
$$

and then use it to calculate the pressure distribution as follows

$$
\nabla \boldsymbol{p}=\rho \boldsymbol{g}-\rho(\boldsymbol{u} \cdot \nabla) \boldsymbol{u}
$$

To simplify this we will expand the super-position of discrete solutions Equation (32) over the whole set of integers $\mathbb{Z}$, then

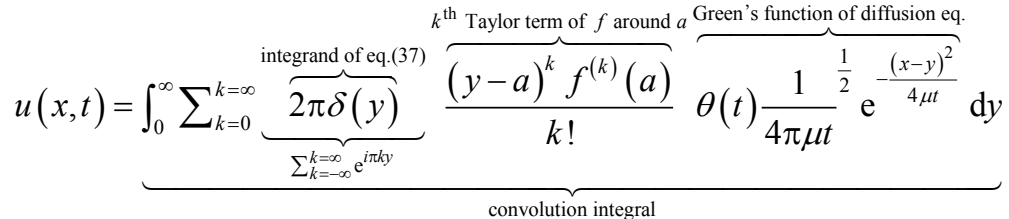

$$
\begin{aligned}
& \rho=1=\sum_{k=0}^{k=\infty} \frac{1}{2} \sqrt{\frac{\pi}{\mu t}} \mathrm{e}^{-\frac{|x|^{2}}{4 \mu t}}-\frac{a^{k} f^{(k)}(a)}{k !} \theta(t)=\frac{1}{2} \sqrt{\frac{\pi}{\mu t}} \mathrm{e}^{-\frac{|x|^{2}}{4 \mu t}} \mathrm{e}^{-a(D f) a} \theta(t)
\end{aligned}
$$

The last function is rescaled along both axes compared to its time-domain version, and is called a "compressed" eigenfunction of the Mellin Transform operator, the corresponding transform pair on the time domain is equivalent with

$$
\pi \sqrt{\frac{1}{4 \pi \mu t}} \mathrm{e}^{\mu t|x|^{2}} \mathrm{e}^{-a(D f) a} \theta(t)
$$

In two dimensions it will be congruent with

$$
\frac{\pi}{4 \mu t} \mathrm{e}^{\mu t\left(|x|^{2}+|y|^{2}\right)} \mathrm{e}^{-a\left(D f_{1}\right) a} \mathrm{e}^{-a\left(D f_{2}\right) a} \theta^{2}(t)
$$

The interested reader may try to derived it, the warped frequency $3 \mathrm{~d}$ pressure distribution in Cartesian coordinates would be then, taking

$$
g=0
$$

out of Equation (54) and disregarding the terms $\mathrm{e}^{-a\left(D f_{j}\right) a}$ for $j=1,2,3$ and the Heaviside time restriction in the different directions,

$$
\begin{aligned}
& \left(\frac{1}{2} \frac{|y| \sqrt{\pi}}{\mu^{2 \frac{1}{2}} t^{2 \frac{1}{2}}} k \operatorname{Erf}\left(\frac{|x|}{\sqrt{4 \mu t}}\right) \exp \left(-\frac{|y|^{2}}{4 \mu t}\right) \exp \left(-\frac{|z|^{2}}{2 \mu t}\right)-\frac{1}{2} \frac{|z| \sqrt{\pi}}{\mu^{2 \frac{1}{2}} t^{2 \frac{1}{2}}} j \operatorname{Erf}\left(\frac{|x|}{\sqrt{4 \mu t}}\right) \exp \left(-\frac{|y|^{2}}{2 \mu t}\right) \exp \left(-\frac{|z|^{2}}{2 \mu t}\right)\right. \\
& \left(\begin{array}{c}
P_{x} \\
P_{y} \\
P_{z}
\end{array}\right)=-\rho\left(\frac{1}{2} \frac{|x| \sqrt{\pi}}{\mu^{2 \frac{1}{2}} t^{2 \frac{1}{2}}} k \operatorname{Erf}\left(\frac{|y|}{\sqrt{4 \mu t}}\right) \exp \left(-\frac{|z|^{2}}{4 \mu t}\right) \exp \left(-\frac{|x|^{2}}{2 \mu t}\right)+\frac{1}{2} \frac{|z| \sqrt{\pi}}{\mu^{2 \frac{1}{2}} t^{2 \frac{1}{2}}} i \operatorname{Erf}\left(\frac{|y|}{\sqrt{4 \mu t}}\right) \exp \left(-\frac{|z|^{2}}{4 \mu t}\right) \exp \left(-\frac{|x|^{2}}{2 \mu t}\right)\right. \\
& \left(\frac{1}{2} \frac{|x| \sqrt{\pi}}{\mu^{2 \frac{1}{2}} t^{2 \frac{1}{2}}} j \operatorname{Erf}\left(\frac{|z|}{\sqrt{4 \mu t}}\right) \exp \left(-\frac{|x|^{2}}{4 \mu t}\right) \exp \left(-\frac{|y|^{2}}{2 \mu t}\right)-\frac{1}{2} \frac{|y| \sqrt{\pi}}{\mu^{2 \frac{1}{2}} t^{2 \frac{1}{2}}} i \operatorname{Erf}\left(\frac{|z|}{\sqrt{4 \mu t}}\right) \exp \left(-\frac{|x|^{2}}{2 \mu t}\right) \exp \left(-\frac{|y|^{2}}{4 \mu t}\right)\right.
\end{aligned}
$$




$$
\text { using } \begin{aligned}
& u_{k}(x, y, t)=\frac{k}{\mu t} \exp \left(-\frac{|x|^{2}}{4 \mu t}\right) \exp \left(-\frac{|y|^{2}}{4 \mu t}\right), \\
& u_{i}(y, z, t)=\frac{i}{\mu t} \exp \left(-\frac{|y|^{2}}{4 \mu t}\right) \exp \left(-\frac{|z|^{2}}{4 \mu t}\right) \text { etc }
\end{aligned}
$$

based on an $i, j, k$ indexed classical Hamilton'ian unit vectors with magnitude $\sqrt{-1}$ where $i^{2}=j^{2}=k^{2}=i j k=-1$ and

$$
i j=k, \quad j k=i, k i=j, i j=-j i, j k=-k j, k i=-i k \text { and where } \operatorname{Erf}(x) \text { the }
$$
Error function is, defined as

$$
\operatorname{Erf}(z)=\frac{2}{\sqrt{\pi}} \int_{0}^{z} \mathrm{e}^{-t^{2}} \mathrm{~d} t
$$

On the edge of the domain the following is true for the normal vectors

$$
\frac{\partial u}{\partial n}=\frac{\partial u_{k}}{\partial z} \cong u(x+k)-u(x)=0 \text { etc. }
$$

Please do see Figure A3. Figure A3 does show the contour graph of the warped frequency distribution in 3 dimensions.

The total energy of each wave system is equal on both the time and frequency domain [6] [7]. In symbols,

$$
\iint_{-\infty}^{\infty} P(t, f) \partial t \partial f=\int_{-\infty}^{\infty}|\mathcal{X}(f)|^{2} \partial f=\int_{-\infty}^{\infty}|x(t)|^{2} \partial t=\mathcal{E}^{2}
$$

with above the expression for the Wigner-Ville Distribution function ( a class of dual energy distributions), and below, the integrand [is equivalent to the Ambiguity Function] as defined in [3] [6] [7]

$$
P(t, f)=f \int_{\mathbb{R}} \mathrm{e}^{-2 \pi i u f t} \frac{u^{2 r+2}}{2 \sinh \frac{u}{2}} \mathcal{X}\left(\frac{f u \mathrm{e}^{\frac{-u}{2}}}{2 \sinh \frac{u}{2}}\right) \mathcal{X}^{*}\left(\frac{f u \mathrm{e}^{\frac{u}{2}}}{2 \sinh \frac{u}{2}}\right) \mathrm{d} u
$$

$\mathcal{X}(f)$ is the analytical signal on the frequency domain and its Fourier Transform inverse $x(t)$ on the time domain and $\mathcal{E}^{2}$ the total (kinetic) energy of the system and $r$ is a warp parameter that may be set $-\frac{1}{2}$ to yield a factor

$$
\frac{u \mathrm{e}^{\frac{u}{2}}}{\mathrm{e}^{u}-1}
$$

in the integrand and what may be calculated with the Residue theorem. Expression (65) will yield an unbounded wave energy-level of $\mathcal{E}^{2}=\int_{-\infty}^{\infty}|u(\mu)|^{2} \partial \mu$ for every component with $\zeta(1)=\infty$.

The Wigner-Ville time-frequency distribution function is a unitary affine function that suffices the following equation

$$
\int_{-\infty}^{\infty} \mathrm{d} t \int_{0}^{\infty} P(t, f) \delta\left(t-\xi-\frac{\beta}{f}\right) f^{2 r} \mathrm{~d} f=\left|\mathcal{M}_{f}^{\xi} \mathcal{X}(\beta)\right|^{2}
$$

with $\mathcal{M}_{f}^{\xi}[\mathcal{X}](\beta)=\int_{0}^{\infty} \mathcal{X}(f) \mathrm{e}^{2 \pi i \xi f} f^{2 \pi i \beta+r} \mathrm{~d} f \quad(12)$.

The total wave energy does inherit the known singularities within the Dirichlet series from the components of the distribution function of $u(x)$ what 


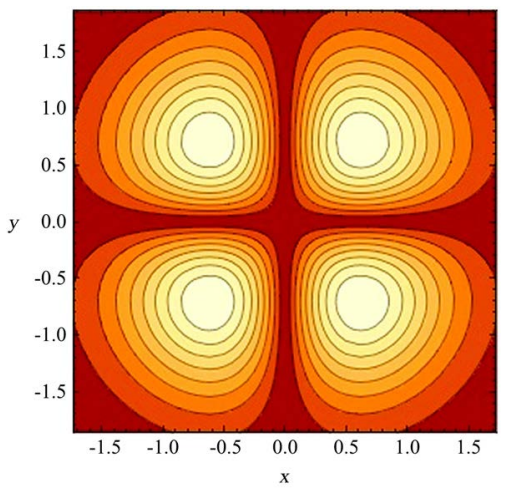

Figure A3. Contour of 3d warped pressure distribution.

makes it also unbounded.

The last maximum value of the total energy is also supported by the fact that the Wigner-Ville Distribution function definition also does have a factor of the hyperbolic cosecant with half argument in its integrand (defined on $C^{\infty}(\mathbb{C} \backslash\{2 \pi \mathbb{Z}\})$, with poles at $\left\{2 \pi i\{k\}_{k \in \mathbb{Z}}\right\}$ what may lead to variants of the improper Riemann contour integral [1]

$$
2 \sin (\pi s) \Gamma(s) \varsigma(s)=i \int_{\infty}^{\infty} \frac{(-x)^{s-1}}{\mathrm{e}^{x}-1} \mathrm{~d} x=i \oint \frac{(-x)^{s-1}}{\mathrm{e}^{x}-1} \mathrm{~d} x
$$

Below schematically, the application of the presented formulas for solving up the Laplace equation in cylindrical coordinates in an analytic signal analyzing model used by [6]. The constructed transformation is scale invariance:

\section{An analytic signal analyzing model}

$$
\begin{aligned}
& \mathcal{X}(f) \text { : } \\
& \zeta\left(-2 \pi i \alpha+\frac{1}{2}\right)=\sum_{|x|=1}^{|x|=\infty}|x|^{2 \pi i \alpha-\frac{1}{2}} \\
& \stackrel{U_{\mathcal{S}}^{s, r}:}{\rightarrow} \\
& U_{f}^{\xi, r} \mathcal{X}(f)=a^{r+1} \mathrm{e}^{-2 \pi i \xi(1-a) f} \mathcal{X}(a f): \\
& \sum_{|x|=1}^{|x|=\infty} \mathrm{e}^{-2 \pi i \alpha|x|} \\
& \text { (warping operator, } r=-\frac{1}{2}, \xi=0 \text {, } \\
& \left.a=\mathrm{e}^{-x} ; \quad U_{x}^{\xi=0, r=-\frac{1}{2}}=U_{x}\right) \\
& \mathcal{M}_{f}^{\xi}[\mathcal{X}(f)]=\mathcal{F} U_{n}^{\xi, r}[\mathcal{X}(f)]: \\
& \mathcal{F} \sum_{|x|=1}^{|x|=\infty} \mathrm{e}^{-2 \pi i \alpha|x|}=\sum_{|x|=1}^{|x|=\infty} \delta(|x|-\alpha) \\
& \mathcal{M}_{f}^{\xi}\left[U_{x}^{\xi, r} \mathcal{X}\right](\beta) \\
& =a^{-2 \pi i \beta} \mathcal{M}_{f}^{\xi}[\mathcal{X}](\mu) \cdot \int_{0}^{\infty} \sum_{|x|=1}^{|x|=\infty} \mathrm{e}^{-2 \pi i \beta|x|}|x|^{\mu-1} \mathrm{~d} x \\
& =\int_{0}^{\infty} \sum_{|x|=1}^{|x|=\infty} \delta(|x|-\beta)|x|^{\mu-1} \mathrm{~d} x \\
& =\zeta(1-\mu) \\
& \int_{-\infty}^{\infty} \sum_{|f|=1}^{|f|=\infty}|f|^{-\alpha} \mathrm{e}^{-2 \pi i(\xi+\beta) f} f^{\mu} \mathrm{d} f \\
& \text { Transform }=2 \pi i^{-\mu} \sum_{|\xi|=1}^{|\xi|=\infty} \frac{\Gamma(\mu+\alpha)}{\Gamma(\alpha)}|\xi+\beta|^{-\mu-\alpha} \\
& =2 \pi i^{-\mu} \frac{\Gamma(\mu+\alpha)}{\Gamma(\alpha)}\left\{\varsigma(\mu+\alpha, \beta)-|\beta|^{-\mu-\alpha}\right\}
\end{aligned}
$$


Out of the general solution is it clear that the distribution functions for $u(x)$ and $p(x)$ on the warped frequency domain do inherit the known singularities of the Dirichlet series, naturally depending on the begin-conditions which may cause shifts in singularities at $\{1\}$, removable singularities at

$$
-\frac{2 \pi \mathbb{Z}}{\log p} i+\frac{\log a(p)}{\log p}
$$

with $p$ a prime number as a consequence of the Euler denomination of the Dirichlet series $\left(a\left(\mathbb{N}^{+}\right)=1\right.$ for the Riemann Zeta function) in the form of the product formula

$$
\sum_{n=1}^{n=\infty} a(n) n^{-s}=\prod_{p \text { prime }} \frac{1}{1-a(p) p^{-s}}
$$

only for $a(n)$ is totally multiplicative [17]. Solving up the zeros of the denominator in the last expression

$$
\mathrm{e}^{2 \pi i \mathbb{Z}}-a(p) p^{-s}=0
$$

does result in the mentioned complex removable singularities. So may be said that the distribution functions $u(x)$ and $p(x)$ are defined on $C^{\infty}(\mathbb{C} \backslash\{\mathbb{Z}\})$ for each component.

The begin-conditions may also dictate the periodicity of the general solution. There exists no general solution that is independent of the begin-solutions, since the solution is constructed out of the begin-conditions. The begin-conditions may cause singularities or may annihilate them, depending on the initial problem and its basic solution. The basic solution of the Laplace equation in cylindrical coordinates, which is the solution core for both the velocity and pressure frequency distributions (in the incompressible case) for the Navier Stokes equations in $2 \mathrm{~d}$ and to certain extent also for the $3 \mathrm{~d}$ version, does contain and consequently inherits the singularities observed in the cited improper Riemann contour integral in particular or from the Dirichlet series in general. The input signal

$$
\mathcal{X}(f)=\sum_{1}^{\infty} f^{\alpha}
$$

of the used model may manifest or cause singularities on both time and warped frequency domains with both the summation parameter indexed by $f$ (hyperbolas) and by $\alpha$ (polynomials). On the time domain the signal may experience singularities due to the vast powers that are used that may be unpractical for most commercial available micro-processors to compute [for example when $f$ is chosen as the series of orthogonal polynomials like the Hermite polynomials or due to the known singularities of the Zeta function. On the warped frequency domain the singularities are due to the properties of the formed Dirichlet series output signals for both cases.

Interesting to note is that the so-called blow-up time within the numeric schemes may manifest whenever the numerical pivoting method strikes a known singularity of the general solution.

When the analytical signal condition is dropped or when negative discrete so- 
lutions of the Laplace equation (read the momentum equation) are allowed, the motion solution of the $3 \mathrm{~d}$ Navier Stokes equations for the viscid incompressible flow will become a (multi-dimensional) Gaussian function with no singularities on neither the time domain nor the warped frequency domain.

\section{Appendix B}

The Riemann Zeta Function represented as a Dirichlet Transform of discrete super-position of second order ODEs solutions.

The confluent hyper geometric functions are solutions to the Weber differential equations, obtained after separation of variables when decomposing the Laplacian in cylindrical coordinates;

$$
\left\{\begin{array}{l}
\frac{\partial u^{2}}{\partial x^{2}}-\left(c+k^{2} x^{2}\right) u=0 \\
\frac{\partial v^{2}}{\partial y^{2}}-\left(c-k^{2} x^{2}\right) v=0
\end{array}\right.
$$

The substitution $u=w \cdot \exp \left(-\frac{x^{2}}{4}\right)$ converts the weber equation to

$$
\frac{\partial w^{2}}{\partial x^{2}}-x \frac{\partial w}{\partial x}+v w=0
$$

with solution $u=H_{v}(x) \cdot \exp \left(-\frac{x^{2}}{4}\right)$, where $H_{v}(x)$ is the Hermite polynomial.

In general the solutions of the second order ordinary confluent hypergeometric d.e.

$$
x \frac{\partial w^{2}}{\partial x^{2}}+(c-x) \frac{\partial w}{\partial x}-a w=0
$$

are of the form

$$
w=b_{11} F_{1}(a ; c ; x)+b_{2} U(a, c, x)
$$

and are called the confluent hypergeometric function of the first and second kind respectively. The first kind version is also denoted as $M(a, c, x)$ or $\Phi(a ; c ; x)$ with

$$
{ }_{1} F_{1}(a ; c ; x)=\sum_{n=0}^{n=\infty} \frac{a_{(n)} z^{n}}{c_{(n)} n !}
$$

and $a_{(n)}$ the Pochhammer symbol defined as

$$
a_{(n)}=\frac{\Gamma(a+n)}{\Gamma(a)}=a(a+1) \cdots(a+n-1)
$$

An integral representation for $U(a, b, z)$ is

$$
U(a, b, z)=\frac{1}{\Gamma(a)} \int_{0}^{\infty} \mathrm{e}^{-z \cdot t} t^{a-1}(1+t)^{b-a-1} \mathrm{~d} t
$$

[18] [19]. 
The confluent hypergeometric function of the second kind, can be transposed to the improper contour integral

$$
i \oint \frac{(-x)^{s-1}}{\mathrm{e}^{x}-1} \mathrm{~d} x
$$

by choosing $b=a+1$, and $a=s$. Namely

$$
\begin{gathered}
U(s, s+1, n)=\frac{1}{\Gamma(s)} \int_{0}^{\infty} \mathrm{e}^{-n \cdot t} t^{s-1} \mathrm{~d} t \\
U(s, s+1, n)=n^{-s}
\end{gathered}
$$

Taking an infinitely summation at both sides of the latter expression and substituting for $n n^{*} n$ and for $s \frac{s}{2}$ will $\varsigma(s)$ emerge as super-position of the solutions, namely

$$
\sum_{n=1}^{n=\infty} U\left(\frac{s}{2}, \frac{s}{2}+1, n^{2}\right)=\varsigma(s)
$$

If we take $\frac{n^{2}}{2}$ instead of $n^{2}$ both sides as argument then the following expression will arise

$$
\sum_{n=1}^{n=\infty} U\left(\frac{s}{2}, \frac{s}{2}+1, \frac{n^{2}}{2}\right)=2^{\frac{s}{2}} \varsigma(s)
$$

$$
D_{v}(z)=2^{\frac{v}{2}} \exp \left(-\frac{z^{2}}{4}\right) U\left(-\frac{1}{2} v, p+\frac{1}{2}, \frac{z^{2}}{2}\right) \text { is a solution to the Weber d.e. }
$$

with $p$ an integer and equal to null. $D_{-v-1}(i \cdot z)$ is the other linear independent solution of Weber d.e.

$$
\frac{\partial y^{2}}{\partial z^{2}}+\left(v+\frac{1}{2}-\frac{1}{4} z^{2}\right) y(z)=0
$$

With substitution $y=u \mathrm{e}^{\frac{-x^{2}}{4}}$ converts the Weber d.e. to $\frac{\partial u^{2}}{\partial x^{2}}+x \frac{\partial u}{\partial x}-v u=0$ with solution $y=\mathrm{e}^{\frac{-x^{2}}{4}} H_{v}(z)$.

$$
\begin{aligned}
D_{n}(z)=2^{\frac{-n}{2}} \exp \left(-\frac{z^{2}}{4}\right) H_{n}\left(\frac{z}{\sqrt{2}}\right) & =\exp \left(-\frac{z^{2}}{4}\right) H_{e_{n}}(z) \text { with } \\
H_{n}(z) & =2^{n} U\left(\frac{-n}{2}, p+\frac{1}{2}, z^{2}\right)
\end{aligned}
$$

the Hermite polynomial and $H_{e_{n}}(z)$ the modified Hermite polynomial.

The modified Hermite polynomial is defined as

$$
H_{e_{n}}(z)=2^{\frac{-n}{2}} H_{n}\left(\frac{z}{\sqrt{2}}\right)=2^{\frac{n}{2}} U\left(\frac{-n}{2}, p+\frac{1}{2}, \frac{z^{2}}{2}\right)
$$

If we denote $p+\frac{1}{2}$ by $-\frac{n}{2}+1$ and compare it to the found expression of $\varsigma(s)$ i.e. 


$$
\varsigma(s)=2^{+\frac{s}{2}} \sum_{n=1}^{n=\infty} U\left(\frac{s}{2}, \frac{s}{2}+1, \frac{n^{2}}{2}\right)
$$

and substituting $s$ by $-s$ yields,

$$
\begin{aligned}
& \varsigma(-s)=\overbrace{2^{-\frac{s}{2}} \sum_{n=1}^{n=\infty} U\left(-\frac{s}{2},-\frac{s}{2}+1, \frac{n^{2}}{2}\right)}^{\sum_{n=1}^{n=\infty} n^{s}} \\
& \tilde{H}_{e_{n}}(z)=\underbrace{2^{\frac{n}{2}} U(\underbrace{\frac{-n}{2}, p+\frac{1}{2}, \frac{z^{2}}{2}}_{-\frac{n}{2}+1})}_{z^{n}}
\end{aligned}
$$

If we interchange the parameters $z$ and $n$ with each other for $\tilde{H}_{e_{n}}(z)$, and superposition all the elements $\tilde{H}_{e_{n}}(n)$ with each other will the expression of $\varsigma(-s)$ emerge as above. The generalized Laguerre polynomials can be represented by

$$
u=L_{-n}^{a}(x)=\frac{(-1)^{-n}}{(-n) !} U(n, a+1, x)
$$

and are related to the Riemann Zeta function as described above. A known transform operator [12] that can transpose polynomial expressions into Dirichlet series is the Dirichlet transform

$$
D_{s} f(x)=\frac{1}{\Gamma(s)} \int_{0}^{\infty} z^{s-1} f\left(x \mathrm{e}^{-z}\right) \mathrm{d} z
$$

that transforms the ordinary generating functions

$$
\begin{gathered}
f_{1}(x)=\sum_{n=1}^{n=\infty} a_{n} x^{n} \\
\text { (OGF) to } f_{4}(x)=\sum_{n=1}^{n=\infty} a_{n} n^{-x}
\end{gathered}
$$

This transform is discussed in the chapter of combinatorics of [15] p. 177 and deals primarily with size conditions of $x \cong|X|=n \in \mathbb{N}$ of the permutations $f(x)=\sum_{k} f(x, k)$ with auxiliary parameter variable $k$.

The Dirichlet series with

$$
f_{4}(s)=\left.D_{s} f_{1}(x)\right|_{x=1}
$$

where it may be used in conjunction with the Laplace-Borel Transform first, as intermediary aid, to first convert the Exponential Generating Functions (EGF)

$$
f_{2}(x)=\sum_{n=1}^{n=\infty} a_{n} \frac{x^{n}}{n !}
$$

into the OGF form what may be the case for the confluent hyper-geometric functions of the first kind. The Laplace-Borel Transform is defined as

$$
\mathcal{L} f_{1}(x)=\int_{0}^{\infty} f_{2}(t x) \mathrm{e}^{t-1} \mathrm{~d} t
$$

In [20] Vojta mentioned about the cannibalistic feature of the Mellin Trans- 
form with regard to the Laplace Transform and "many other functions" what may validate the two step approach to calculate the Dirichlet Transform result for the generalized Laguerre polynomials.

Table B1 shows the study of several second order ODEs with regard to their solutions on the time domain, and the improper summation of their Dirichlet transforms on the warped frequency domain. The transform of the solution double argument functions $f_{n}(x)$ entails a variable swap between the domain variables $n \in \mathbb{N}$ and $x \in \mathbb{C}$.

$$
K_{\frac{1}{2}-n} \mathrm{e}^{\frac{x}{2}} x^{\frac{1}{2}-n}=U(n, 2 n, x) \text { is one of the independent solutions of the confluent }
$$

hypergeometric differential equation. The row elements in the Table B2 are enclosed in the integer sequence OIES A113025 [23] and the summation of the diagonal elements $a_{n}=\sum_{n=1}^{n=\infty} \sum_{k=0}^{n} a_{n, k}$ are the Dirichlet coefficients of $f_{4}(x)=D_{s} f_{1}(x)$.

Table B1. Second order ODEs (Ordinary Differential Equations) and their solutions on time and warped frequency domains.

\begin{tabular}{|c|c|c|c|c|}
\hline Type & D.e. & Solution & $\begin{array}{l}\text { Dirichlet Transform of } \\
\text { superposition of (discretized) } \\
\text { solutions with explanation }\end{array}$ & $\begin{array}{l}\text { Dirichlet Transform of } \\
\text { superposition of } \\
\text { (discretized) solutions }\end{array}$ \\
\hline EULER & $\begin{array}{l}x^{2} \frac{\partial u^{2}}{\partial x^{2}}+x \alpha \frac{\partial u}{\partial x} \\
+\beta u=0\end{array}$ & $\begin{array}{l}u(x)=x^{\lambda} \cos (\mu \ln x)+i x^{\lambda} \sin (\mu \ln x) \\
=x^{\lambda} \exp (i \mu \ln x)\end{array}$ & $\begin{array}{l}D_{s} \sum_{\mu=1}^{\mu=\infty} \underbrace{x^{\lambda} \exp (i \mu \ln x)}_{x^{\lambda+1 \mu}} \\
=\frac{1}{\Gamma(s)} \mathcal{M}_{x} \sum_{\mu=1}^{\mu=\infty} \mathrm{e}^{-\lambda x} \exp \left(i \mu \ln \mathrm{e}^{-x}\right) \\
=(-i)^{-s} \varsigma(s,-i \lambda)=\Phi(1, s,-i \lambda) i^{s} \\
(99) \\
\Phi \text { is Lerch Transcedent function }\end{array}$ & $\begin{array}{l}(-i)^{-s} \varsigma(s,-i \lambda) . \\
\varsigma(s, \lambda)=\sum_{n=0}^{n=\infty} \frac{1}{(n+\lambda)^{s}} \quad(100) \\
\text { is the Hurwitz Zeta function as } \\
\text { generalization of } \varsigma(s) \text { with } \\
\varsigma(s, 1)=\varsigma(s) .\end{array}$ \\
\hline $\begin{array}{l}\text { variant } \\
\text { modified } \\
\text { Hermite }\end{array}$ & $\begin{array}{l}\frac{\partial u^{2}}{\partial x^{2}}-\left(\frac{n}{2}+x-1\right) \frac{\partial u}{\partial x} \\
+n u=0 \\
(101)\end{array}$ & $\tilde{H}_{e_{n}}(z)=U(\frac{-n}{2}, \underbrace{p+\frac{1}{2}}_{-\frac{n}{2}+1}, \frac{z^{2}}{2})$ & $\varsigma(-S)=\overbrace{2^{\frac{s}{2}} \sum_{n=1}^{n=\infty} U\left(-\frac{s}{2},-\frac{s}{2}+1, \frac{n^{2}}{2}\right)}^{2^{\frac{s}{2}-\frac{s}{2}} \sum_{n=1}^{n=0} n^{s}}$ & $\sum_{n=1}^{n=\infty} D_{s} \tilde{H}_{e_{n}}(z)=\varsigma(-S)$ \\
\hline $\begin{array}{l}\text { Confluent } \\
\text { hypergeometric }\end{array}$ & $\begin{array}{l}\frac{\partial u^{2}}{\partial x^{2}}+(c-x) \frac{\partial u}{\partial x} \\
-a u=0\end{array}$ & $\begin{array}{l}u=b_{11} F_{1}(a ; c ; x)+b_{2} U(a, c, x) ; \\
\text { Special cases: } \\
{ }_{1} F_{1}(n, n+1, x) \\
\sim \Gamma(1+n) \Gamma(1-n) L_{-n}^{n}(x) \\
\text { with } L_{i}^{n}(x) \text { the associated Laguerre } \\
\text { polynomials [21] } \\
U(n, n+1, x) \sim x^{-n} \quad(107) \\
U(n, 2 n, x) \\
\sim \frac{\mathrm{e}^{\frac{x}{2}}}{\sqrt{\pi}} x^{\frac{1}{2}-n} K_{n-\frac{1}{2}}\left(\frac{x}{2}\right) \sim a_{n} x^{-n} \quad(109) \\
{ }_{1} F_{1}(n, 2 n, x) \\
\sim \frac{\mathrm{e}^{\frac{x}{2}}}{\sqrt{\pi}} \frac{x^{\frac{1}{2}}}{4} \Gamma\left(n+\frac{1}{2}\right) I_{n-\frac{1}{2}}\left(\frac{x}{2}\right) \quad(113) \\
\sim \frac{n_{(n)}}{n_{(2 n)}} \frac{x^{n}}{n !} \\
; \text { With } \Gamma, \Gamma(; ;), I, K \text {, resp. the gamma }\end{array}$ & $\begin{array}{l}\Gamma(1+s) \Gamma(1-s) L_{-x}^{x}(n) \\
=\Gamma(1+s) \Gamma(1-s) \frac{-1^{-s} U(s, s+1, n)}{(-s) !} \\
=-1^{-s} U(s, s+1, n) \Gamma(1+s) \\
(104) \\
-1^{-s} \Gamma(s+1) \varsigma(s) \quad(105), \text { since } \\
=\sum_{n=1}^{n=\infty}{ }_{1} F_{1}(s, s+1, n) \\
\varsigma(s)=\sum_{n=1}^{n=\infty} U(s, s+1, n) . \\
\varphi(s)=\sum_{n=1}^{n=\infty} a_{n} n^{-s} \quad(110) ; \\
a_{n}=\sum_{n=1}^{n=\infty} \sum_{k=0}^{n} a_{n, k} \quad(111) \text { with } \\
a_{n, k} \quad \text { OIES A113025. See Table B2. } \\
\Gamma(s) \varphi(s)=\Gamma(s) \sum_{n=1}^{n=\infty} a_{n} n^{-s \quad(114) ;} \\
a_{n}=\frac{n_{(n)}}{n_{(2 n)}}(115) ; \\
\varphi(s)=\sum_{n=1}^{n=\infty} a_{n} n^{-s} \quad(116) ;\end{array}$ & $\begin{array}{l}D_{s} \sum_{n=1}^{n=\infty} F_{1}(n, n+1, x) \\
=-1^{-s} \Gamma(s+1) \varsigma(s) \\
D_{s} \sum_{n=1}^{n=\infty} U(n, n+1, x)=\varsigma(s) \\
(108) \\
D_{s} \sum_{n=1}^{n=\infty} U(n, 2 n, x)=\varphi(s) \\
(112) \\
\varphi(s)=\sum_{n=1}^{n=\infty} a_{n} n^{-s} \quad(110) \\
D_{s} \sum_{n=1}^{n=\infty} F_{1}(n, 2 n, x)=\varphi(s) \Gamma(s) \\
(116) \\
\Gamma(s) \varphi(s)=\Gamma(s) \sum_{n=1}^{n=\infty} a_{n} n^{-s} \\
(114) \\
D_{s} \sum_{n=1}^{n=\infty} U(n, 2 n, x)=\varphi(s) \\
(118)\end{array}$ \\
\hline
\end{tabular}




\section{Continued}

Generalized or associated Laguerre

Bessel $\frac{\partial u^{2}}{\partial x^{2}}+(a+1-x) \frac{\partial u}{\partial x}$ $+n u=0$

(119);

function, the generalized gamma $a_{n}=\sum_{n=1}^{n=\infty} \sum_{k=0}^{n} a_{n, k}(117) ;$

function, the modified Bessel function
of first and second kinds, $n$ the with $a_{n, k}$ OIES A001497

Pochhammer symbol

$U(n, 2 n, x)=2^{-n} \theta_{-n}\left(\frac{x}{2}\right) \quad(115) ; \theta$ is

a (reverse) Bessel polynomial [22]

when $n$ is a non-negative integer

$u=L_{n}^{a}(x)=\frac{(-1)^{n}}{n !} U(-n, a+1, x)$

Step 1: Laplace-Borel

Special cases:

Transformation of

$L_{n}^{a}(x)=\frac{(-1)^{n}}{n !} U(-n,-n+1, x)$

$\sum_{n=1}^{n=\infty} \frac{(-1)^{n}}{n !} x^{n}=\sum_{n=1}^{n=\infty}(-x)^{n}$

Step 2:

$$
\mathrm{EGF} \equiv f_{3}(-x)=\sum_{n=1}^{n=\infty} \frac{(-1)^{n}}{n !} x^{n}
$$

$\sim \frac{(-1)^{n}}{n !} x^{n}$

$D_{s} \sum_{n=1}^{n=\infty}(-x)^{n}=-2^{-s}\left(2^{s}-2\right) \varsigma(s)$

$D_{s} \mathcal{L} f_{3}(-x)=D_{s} \mathcal{L} \sum_{n} L_{n}^{a}(x)$

$=-2^{-s}\left(2^{s}-2\right) \varsigma(s)$

(120)

$\sum_{k=1}^{k=\infty} D_{s}\left(Y_{\frac{1}{2}}(2 \pi k x)+i J_{\frac{1}{2}}(2 \pi k x)\right)$

$=\frac{1}{\Gamma(s)} \sqrt{\frac{1}{2 \pi}} \mathcal{M}_{k}^{\xi}\left[U_{k}^{\xi, r} \sum_{k=1}^{k=\infty} \mathrm{e}^{2 \pi i k x}\right]$

$y=x^{-p}\left[c_{1} J_{\frac{q}{r}}\left(\frac{a}{r} x^{r}\right)+c_{2} Y_{\frac{q}{r}}\left(\frac{a}{r} x^{r}\right)\right] ;$

$\frac{1}{\Gamma(s)} \sqrt{\frac{1}{2 \pi}} \mathcal{M}_{k}^{\xi}\left[\mathrm{e}^{-2 \pi i k x} \sum_{k=1}^{k=\infty} \mathrm{e}^{2 \pi i k x}\right]$

$x^{2} \frac{\partial u^{2}}{\partial x^{2}}+x(2 p+1) \frac{\partial u}{\partial x}$

$J_{\frac{1}{2}}(k x)=\sqrt{\frac{2}{\pi}} \frac{\sin (k x)}{x}$

$+\left(\beta^{2}+x^{2} a^{2 r}\right) y=0$

$Y_{\frac{1}{2}}(k x)=\sqrt{\frac{2}{\pi}} \frac{\cos (k x)}{x}$

$=\frac{1}{\Gamma(s)} \sqrt{\frac{1}{2 \pi}} \sum_{k=1}^{k=\infty} k^{s-1} d k$

$\sum_{k=1}^{k=\infty} D_{s}\left(Y_{\frac{1}{2}}(2 \pi k x)+i J_{\frac{1}{2}}(2 \pi k x)\right)$

$p=\frac{1}{2}, \quad \beta=0, \quad r=1$

$J$ and $Y$ the Bessel function of first and $=\frac{1}{s \Gamma(s)} \sqrt{\frac{1}{2 \pi}} \zeta(-s)$

$=\frac{1}{s \Gamma(s)} \sqrt{\frac{1}{2 \pi}} \zeta(-s)$

$s=2 \pi i \beta-1 ; \quad \xi=0 ; a=\mathrm{e}^{-x} ;$

$r=-2, \quad Z(f)=\mathrm{e}^{2 \pi i k f}, f>0$

Table B2. Progression of the modified Bessel function of the first kind $K_{\frac{1}{2}-n}$.

\begin{tabular}{|c|c|c|c|c|c|c|c|c|c|c|c|c|}
\hline $\begin{array}{c}a_{n, k} \mid n \rightarrow \\
k \downarrow\end{array}$ & $K_{\frac{1}{2}-k}$ & & & & & & & & & & & $\begin{array}{c}\text { Expression for } \\
K_{\frac{1}{2}-k}\end{array}$ \\
\hline$k=0$ & $K_{\frac{1}{2}}$ & & & & & & 1 & & & & & $\sqrt{\pi} \mathrm{e}^{\frac{-x}{2}} x^{-\frac{1}{2}}$ \\
\hline$k=1$ & $K_{-\frac{1}{2}}$ & & & & & 1 & & 2 & & & & $\sqrt{\pi} \mathrm{e}^{\frac{-x}{2}} x^{-\frac{1}{2}}\left(\frac{2}{x}+1\right)$ \\
\hline$k=2$ & $K_{-1 \frac{1}{2}}$ & & & & 1 & & 6 & & 12 & & & $\sqrt{\pi} \mathrm{e}^{\frac{-x}{2}} x^{-\frac{1}{2}}\left(\frac{12}{x^{2}}+\frac{6}{x}+1\right)$ \\
\hline$k=3$ & $K_{-2 \frac{1}{2}}$ & & & 1 & & 12 & & 60 & & 120 & & $\sqrt{\pi} \mathrm{e}^{\frac{-x}{2}} x^{-\frac{1}{2}}\left(\frac{120}{x^{3}}+\frac{60}{x^{2}}+\frac{12}{x}+1\right)$ \\
\hline$k=4$ & $K_{-3 \frac{1}{2}}$ & & 1 & & 20 & & 180 & & 840 & & 1680 & $\sqrt{\pi} \mathrm{e}^{\frac{-x}{2}} x^{-\frac{1}{2}}\left(\frac{1680}{x^{4}}+\frac{840}{x^{3}}+\frac{180}{x^{2}}+\frac{12}{x}+1\right)$ \\
\hline$k=5$ & $K_{-4 \frac{1}{2}}$ & 1 & & 30 & & 420 & & 3360 & & 4120 & 30,240 & $\sqrt{\pi} \mathrm{e}^{\frac{-x}{2}} x^{-\frac{1}{2}}\left(\frac{1680}{x^{4}}+\frac{840}{x^{3}}+\frac{180}{x^{2}}+\frac{12}{x}+1\right)$ \\
\hline & $x^{0}$ & & $x^{-1}$ & & $x^{-2}$ & & $x^{-3}$ & & $x^{-4}$ & & $x^{-5}$ & $\sqrt{\pi} \mathrm{e}^{\frac{-x}{2}} x^{-\frac{1}{2}}\left(\frac{30240}{x^{4}}+\frac{4120}{x^{4}}+\frac{33160}{x^{3}}+\frac{420}{x^{2}}+\frac{30}{x}+1\right)$ \\
\hline
\end{tabular}

